\title{
Contrast inversion in non-contact atomic force microscopy imaging of $\mathbf{C}_{60}$ molecules
}

\author{
Felix Loske, Philipp Rahe and Angelika Kühnle \\ Fachbereich Physik, Universität Osnabrück, Barbarastraße 7, 49076 Osnabrück, Germany \\ E-mail: kuehnle@uos.de
}

Received 4 December 2008, in final form 16 January 2009

Published 10 June 2009

Online at stacks.iop.org/Nano/20/264010

\begin{abstract}
Non-contact atomic force microscopy (NC-AFM) was applied to study $\mathrm{C}_{60}$ molecules on rutile $\mathrm{TiO}_{2}$ (110). Depending on the tip-sample distance, distinctly different molecular contrasts are observed. Systematically decreasing the tip-sample distance results in contrast inversion that is obtained reproducibly on the $\mathrm{C}_{60}$ islands. This change in contrast can be related to frequency shift versus distance $(\mathrm{d} f(z))$ curves at different sample sites, unraveling crossing points in the $\mathrm{d} f(z)$ curves in the attractive regime. We have performed simulations based on a simple Morse potential, which reproduce the experimental results. This combined experimental and simulation study provides insight into the mechanisms responsible for molecular contrast in NC-AFM imaging. Moreover, this work demonstrates the importance of distance-dependent measurements for unambiguously identifying molecular positions within a molecular island using NC-AFM.
\end{abstract}

(Some figures in this article are in colour only in the electronic version)

\section{Introduction}

The concept of using organic molecules as tailor-made building blocks for functional devices has attracted great interest since decades [1]. For the production of molecular devices, however, the spontaneous ordering of molecules on surfaces needs to be explored [2]. In particular, a detailed knowledge of both intermolecular as well as molecule-substrate interactions is required for successfully employing self-assembly techniques in device fabrication. Molecular self-assembly has been studied intensively with scanning tunneling microscopy (STM) on metal surfaces [3]. While STM images the surface electronic structure, the surface force field is probed with noncontact atomic force microscopy (NC-AFM). NC-AFM has been proven to be a powerful and versatile tool for investigating a wide range of surfaces and, in advantage to STM, also dielectric substrates can be probed [4-7]. Compared to STM, however, the understanding of contrast formation in NC-AFM is far less mature. Understanding and interpreting experimental NC-AFM data has been addressed recently investigating both, bare as well as adsorbate-covered surfaces [8-13]. Contrast formation of single $\mathrm{C}_{60}$ molecules has been studied on $\mathrm{Si}(111)-(7 \times 7)$, revealing $\mathrm{C}_{60}$ molecules that are imaged as depressions [14]. In this study it has been suggested that this contrast inversion might originate from a jump of the tip above the $\mathrm{C}_{60}$ molecule, entering into the regime of negative slope in the frequency shift versus distance $(\mathrm{d} f(z))$ curve. Also when imaging extended $\mathrm{C}_{60}$ islands on $\mathrm{Si}(111)-(7 \times 7)$ contrast inversion was observed and assumed to be due to a change in the vibration amplitude compared to the topography signal [15].

Here, we study the interaction between $\mathrm{C}_{60}$ molecules within a $\mathrm{C}_{60}$ island on rutile $\mathrm{TiO}_{2}(110)$ and the scanning probe tip. The contrast formation was studied in dependence on the tip-sample distance. Far away from the sample surface, each $\mathrm{C}_{60}$ molecule is imaged as a bright feature. When decreasing the tip-sample distance, the contrast changes, eventually resulting in $\mathrm{C}_{60}$ molecules imaged as depressions. The resulting relation between measured frequency shift values at defined positions on the molecular island versus the tipsample distance is plotted as $\mathrm{d} f(z)$ curves. Our results reveal crossing points in the $\mathrm{d} f(z)$ curves at different surfaces positions, explaining why contrast inversion is observed on the $\mathrm{C}_{60}$ molecules upon decreasing the tip-sample distance. We have performed simulations based on a simple model system, closely resembling the experimental observations. Comparing these simulations with the experimental results allows for gaining insight into the interaction between $\mathrm{C}_{60}$ molecules and the scanning probe tip. In particular, we can assign the observed change in contrast to crossing points in the attractive 
region of tip-sample interaction, i.e., the observed contrast inversion is not due to entering into the repulsive regime. This study emphasizes the fact that a detailed distance-dependent analysis is usually required for unambiguously identifying molecular positions within a molecular island.

\section{Experimental methods}

Measurements were performed in an ultra-high vacuum (UHV) system described before [16]. We use a VT-AFM 25 from Omicron (Taunusstein, Germany) with modified electronics operated in frequency modulation non-contact atomic force microscopy (FM NC-AFM) mode. All images shown here are taken at room temperature. As probes we use silicon cantilevers (PPP-NCH from Nanosensors, Neuchâtel, Switzerland) with resonance frequencies $f_{0}$ of about $300 \mathrm{kHz}$ and typical quality factors of 30000 . The cantilevers were excited to oscillations with a peak-to-peak amplitude of $20 \mathrm{~nm}$. Tips were $\mathrm{Ar}^{+}$ion sputtered at $2 \mathrm{keV}$ for $5 \mathrm{~min}$ to remove contaminants. To minimize long-range electrostatic interactions, an appropriate bias voltage of approximately $-1 \mathrm{~V}$ was applied to the tip. The compensating voltage was determined via Kelvin probe force spectroscopy [17].

Rutile $\mathrm{TiO}_{2}$ samples were crystals of highest available quality (MTI, Richmond, USA). The surface was cleaned by repeated cycles of $\mathrm{Ar}^{+}$sputtering at $1 \mathrm{keV}$ and annealing at $1100 \mathrm{~K}$. The $\mathrm{C}_{60}$ molecules (purity of $99.95 \%$, MER Corporation, Tuscon, Arizona) were deposited onto the surface by thermal evaporation from a home-made Knudsen cell heated to $500 \mathrm{~K}$. During evaporation, the substrate was held at room temperature. The deposition rate of $0.12 \mathrm{ML} \mathrm{min}^{-1}$ (1 ML equals to $0.2 \mathrm{C}_{60}$ molecules per $\mathrm{TiO}_{2}(110)$ unit cell) was measured with a quartz crystal deposition monitor (Inficon, East Syracuse, USA).

Depending on the distance feedback loop settings, either constant frequency shift ( $z$ contrast) or constant height images (df contrast) can be recorded [18]. When the distance feedback is switched off, true constant height images are obtained. In the present experiments, however, the distance feedback could not be switched off completely as we needed to compensate for sample tilt and thermal drift in $z$-direction. Consequently, all images shown here are taken with slow feedback loop settings in which the vertical movement following the corrugation of the molecules within an island is in the order of $\pm 5 \mathrm{pm}$, resulting in quasi-constant-height mode images, i.e., we present the df contrast. In this work, we discuss distancedependent df curves, thus, presenting $\mathrm{d} f(z)$ curves would be the most direct way of data presentation. However, our experiments require site specificity, which is only provided when lateral drift is negligible. When operated at room temperature, usual AFM setups without special precautions such as atom tracking [19] do not provide high enough drift stability. In order to arrive at meaningful data, we present the measured df values in dependence on the df setpoint. This approach is justified as the tip-sample distance is controlled by the frequency shift setpoint, which represents the average frequency shift of the df images. As we exclusively measure in the attractive regime (will be proven later) the frequency shift setpoint is monotonically dependent on the tip-sample distance. Thus, the df setpoint serves as a measure for the tipsample distance in our experiments. The images are displayed such that bright areas correspond to high attractive interaction while dark corresponds to less attractive or even repulsive interactions [11].

\section{Results and discussion}

\subsection{Experimental results}

On the rutile $\mathrm{TiO}_{2}$ (110) surface $\mathrm{C}_{60}$ molecules are known to arrange in a rhombic superstructure with an angle of $81^{\circ} \pm 1^{\circ}$ and with the $\mathrm{C}_{60}$ molecules lying centered in the troughs formed by the bridging oxygen rows, as shown in figures 1(a) and (b) [16]. Protruding molecular rows are observed on the $\mathrm{C}_{60}$ islands, which are ascribed to domain boundaries originating from stacking faults. The molecules in the domain boundary are directly surrounded by six other $\mathrm{C}_{60}$ molecules that are marked in figure 1(b).

In figure 2(a) a zoom into the marked area from figure 1(a) is depicted, showing a $\mathrm{C}_{60}$ layer with domain boundaries. In the following images (b)-(g) the tip-sample distance was decreased successively by changing the frequency shift setpoint from -10.4 to $-25.1 \mathrm{~Hz}$. When scanning at different frequency shift setpoints, we observe different contrasts on the molecular island. This evolution in contrast was reproducible obtained when retracting the tip afterward. In particular, we do not observe any sign for a tip change, i.e., the observed changes in contrast can be ascribed to the change in tip-sample distance solely. In general, imaging was observed to be rather stable, especially when compared to imaging on bare $\mathrm{TiO}_{2}(110)$. One explanation for these stable imaging conditions could be an initial transfer of $\mathrm{C}_{60}$ molecules to the very end of the tip. All images would then be obtained with a rather stable $\mathrm{C}_{60}$ tip apex.

To follow the steps of change in contrast, three different positions on the molecular island are marked in different colors in the schematic in figure 1(b): position A (triangle) is in the center of the $\mathrm{C}_{60}$ unit cell, position $\mathrm{B}$ (circle) between two nearest-neighbor $\mathrm{C}_{60}$ molecules and position $\mathrm{C}$ (square) on top a $\mathrm{C}_{60}$ molecule. The same positions are also indicated in the NC-AFM images in figures $2(\mathrm{a})-(\mathrm{g})$. For each NCAFM image, the measured frequency shifts at these three positions were averaged over eight unit cells and plotted against the preset frequency shift setpoint for each image (figure 3(a)). The error of this values amounts to $\pm 0.3 \mathrm{~Hz}$ approximately. Within the range of tip-sample distances considered here, the measured frequency shifts represent a monotonically increasing curve. This indicates that we measure in the attractive regime solely [11]. Between the images in figures 2(b) and (c) the tip-sample distance was decreased by changing the frequency shift setpoint from -12.6 to $-15.1 \mathrm{~Hz}$. This results in a less corrugated appearance of the $\mathrm{C}_{60}$ molecules in figure 2(c), as the difference in measured frequency shift at positions $\mathrm{C}$ and $\mathrm{B}$ is reduced. In the image in figure $2(\mathrm{~d})$, taken at a frequency shift setpoint of $-17.1 \mathrm{~Hz}$, the contrast has changed. Now position $B$ between the $C_{60}$ 


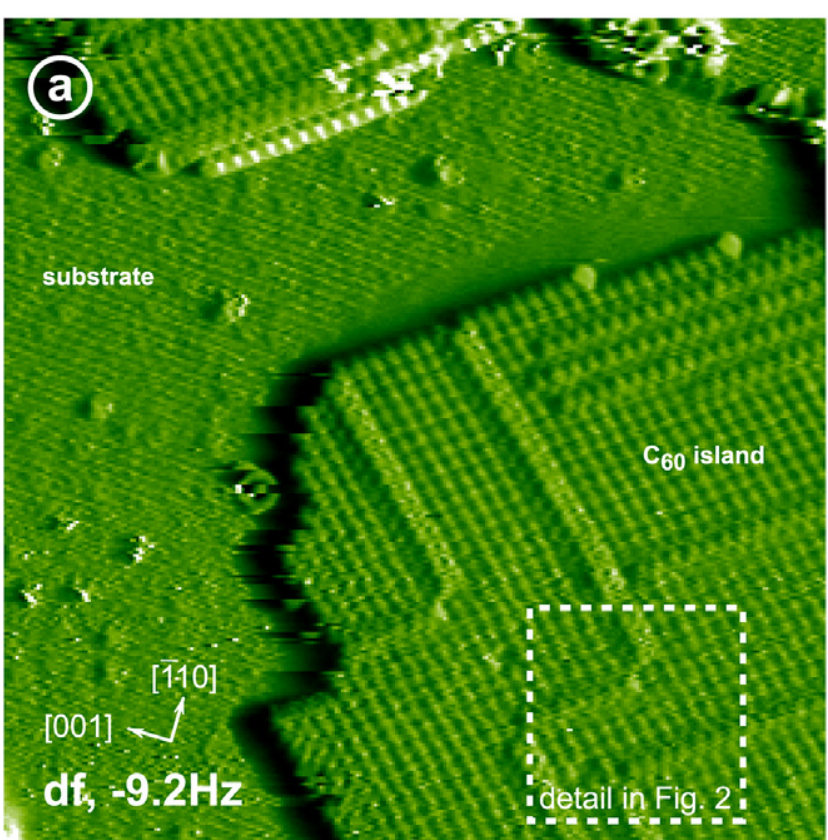

(b)

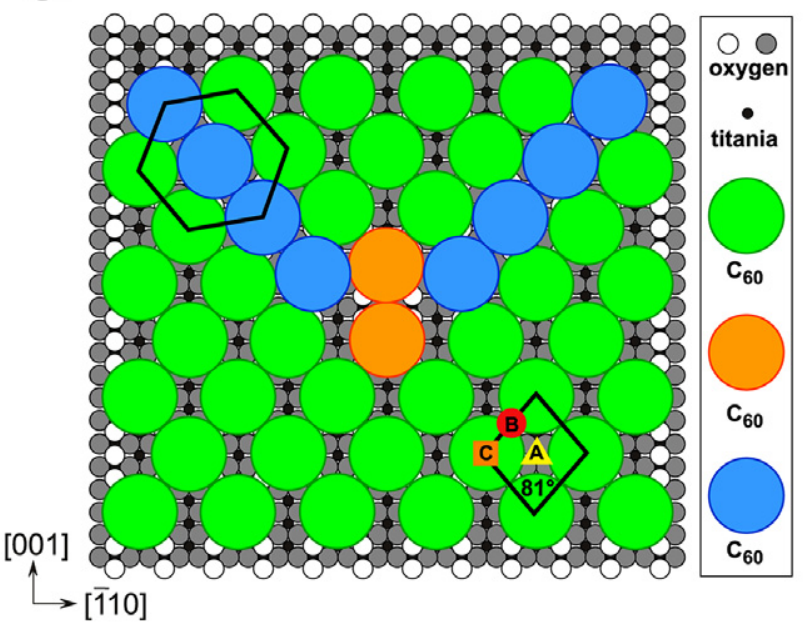

Figure 1. (a) Frequency shift image showing a typical $\mathrm{C}_{60}$ island as reported previously in [16]. Frame size is $48 \mathrm{~nm} \times 48 \mathrm{~nm}$. Backward scan (from right to left) taken at a frequency shift setpoint of

$-9.8 \mathrm{~Hz}$. (b) Schematic of the molecular arrangement of $\mathrm{C}_{60}$ on $\mathrm{TiO}_{2}(110)$ and model of the domain boundary [16].

molecules is brighter than position $\mathrm{C}$ on the $\mathrm{C}_{60}$ molecules, which can also be seen in the crossing of the corresponding curves in figure 3(a). Position A in the center of the unit cell appears, however, still as darkest position. This corresponds to the least attractive interaction at sample position A.

Upon further decreasing the tip-sample distance by changing the frequency shift setpoint to $-19.1 \mathrm{~Hz}$ (figure 2(e)) position $\mathrm{A}$ gets as bright as position $\mathrm{B}$, while position $\mathrm{C}$ on top of a $\mathrm{C}_{60}$ molecules appears darkest. Finally, in figures 2(f) and $(\mathrm{g})$ position $\mathrm{A}$ becomes brightest. Thus, in comparison to figure 2(a) the contrast has inverted completely. In figure 2(a) the order in attractive tip-molecule interaction is A, B, C whereas in figure $2(\mathrm{~g})$ it is $\mathrm{C}, \mathrm{B}, \mathrm{A}$. This is also seen in the curves given in figure 3 (a).
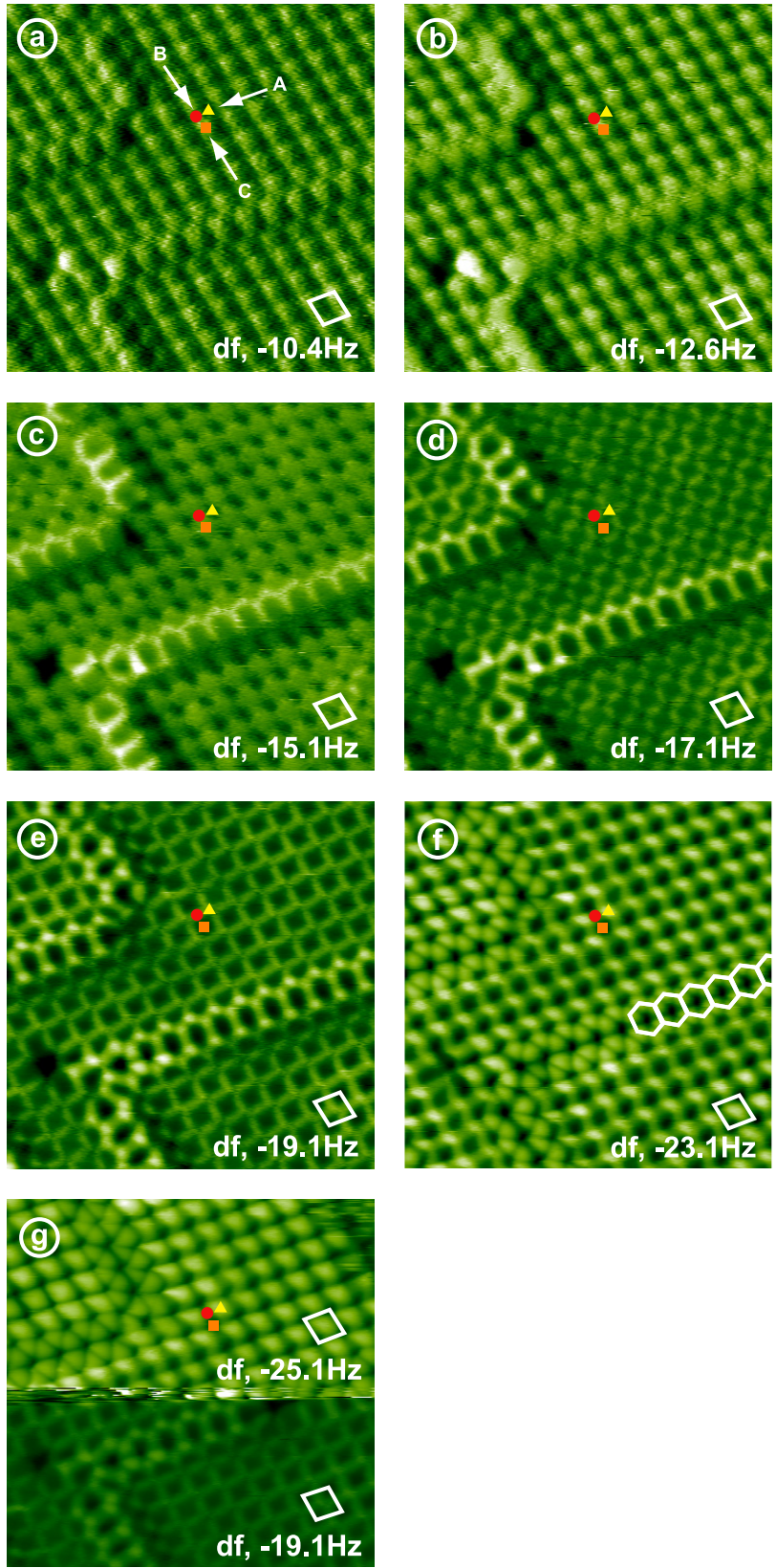

Figure 2. Contrast inversion on a $\mathrm{C}_{60}$ island. Series of quasi-constant-height images upon decreasing the tip sample distance. Frame size is $12 \mathrm{~nm} \times 12 \mathrm{~nm}$. Forward scan (from left to right) images with the frequency shift setpoint noted in the images. The frequency shift setpoint was increased successively from image (a) to (g), inducing a change in imaging contrast. Three positions are marked in every image, corresponding to the positions indicated in the schematic shown in figure 1(b). At these positions the measured frequency shift was analyzed and compiled in figure 3(a), comparable to $\mathrm{d} f(z)$ curves.

The same change in contrast is observed at the domain boundaries. As the boundaries are protruding from the underlying $\mathrm{C}_{60}$ layer [16], the contrast inversion takes place already at larger tip-sample distances, since the tip approaches the protruding features more closely compared to the $\mathrm{C}_{60}$ island. The contrast pattern on top of the domain boundaries shows a hexagonal structure, corresponding to the four directly 

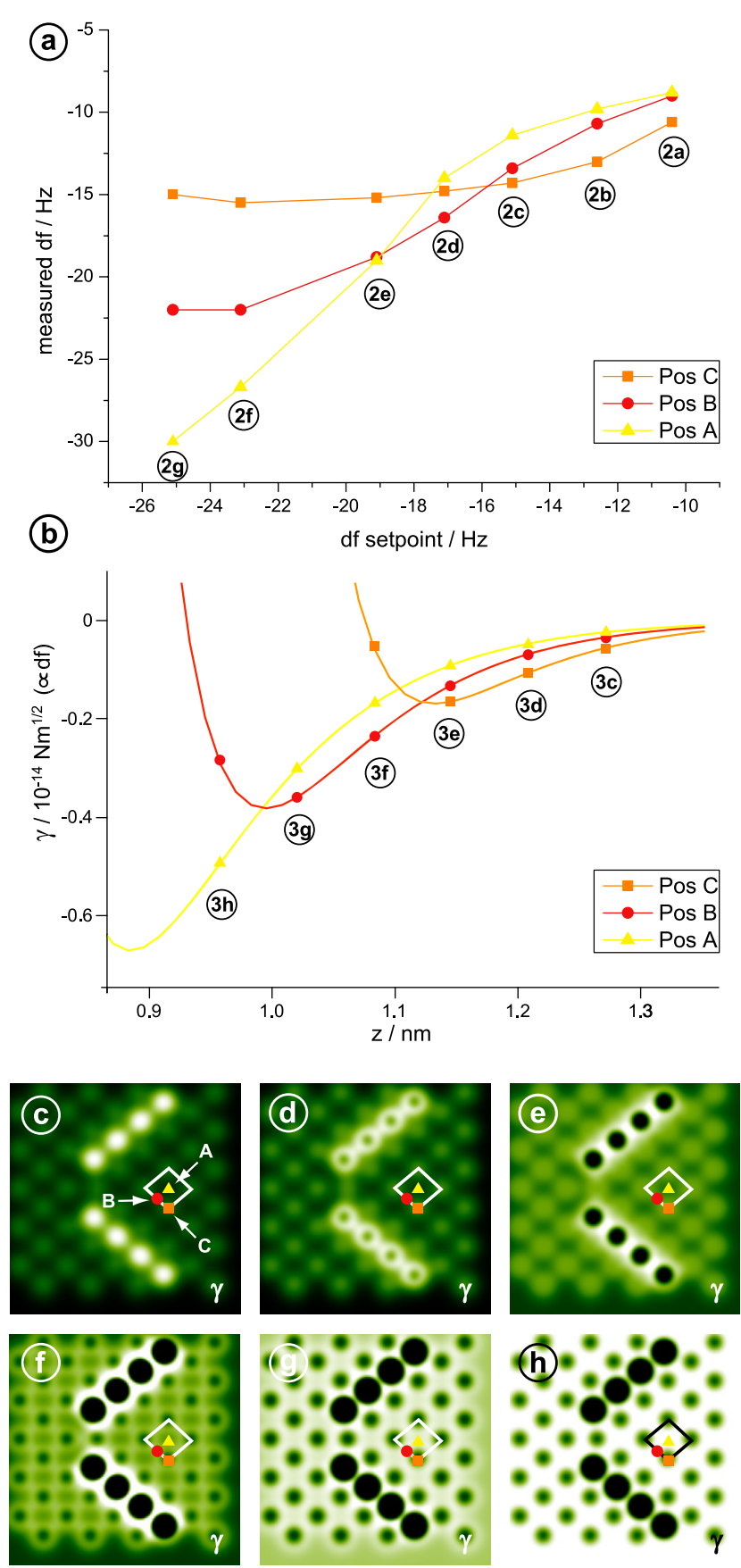

Figure 3. (a) Experimentally obtained frequency shift at defined sample positions plotted against the preset frequency shift setpoints. The error of the displayed frequency shift values amounts to $\pm 0.3 \mathrm{~Hz}$ approximately. The curves are comparable to $\mathrm{d} f(z)$ curves as the frequency shift setpoint is related to the mean height over the surface. (b) Corresponding simulated frequency shift versus distance curves at the same positions on the $\mathrm{C}_{60}$ islands. Note that $\gamma$ is directly proportional to $\mathrm{df}$ as stated in the main text (equation (1)).

(c)-(h) Simulated images showing the normalized frequency shift at different tip-sample distances over a $\mathrm{C}_{60}$ island with a domain boundary.

surrounding $\mathrm{C}_{60}$ molecules of the underlying $\mathrm{C}_{60}$ layer and the two neighboring molecules in the domain boundary.

This hexagonal structure appears not completed, but open at the rear side relative to the fast scan direction (figures 2(c)-(e)). We account this for a feedback effect, which can be clearly seen when comparing forward and backward scans of an image, as shown in figures 4(a) and (b). When considering the forward scan (fast scan direction from left to right) the tip is approaching the left-hand site of the domain boundary very closely. As we scan with slow feedback loop settings, the tip is retracted slowly a few $10 \mathrm{pm}$, resulting in a slightly larger tip-sample distance at the right-hand side of the domain boundary. Therefore, the bright rim is not seen at the right-hand side of the domain boundary. The same effect is observed vice versa in a backward scan, resulting in a hexagonal structure that is open to the left as shown in figure 4(b).

\subsection{Model simulations}

To gain insight into the observed contrast formation and to rule out influences of the feedback loop, we performed simulations using a simple model based on a Morse potential. This potential is used for modeling a pairwise, additive interaction between each single $\mathrm{C}_{60}$ molecule and the probing tip. For the simulations we assume that a single $\mathrm{C}_{60}$ is pinned to the tip. This is motivated by the experimentally observed stable imaging conditions mentioned before, which are rather unique for $\mathrm{C}_{60}$ molecules on $\mathrm{TiO}_{2}(110)$ compared to bare $\mathrm{TiO}_{2}(110)$.

To model the interaction, we use a Morse potential to demonstrate that this contrast mechanism is not necessarily a peculiarity of the $\mathrm{C}_{60}$ system, but may also occur when measuring other substrate-adsorbate systems. Nevertheless, we tested this approach with two other potentials proposed for $\mathrm{C}_{60}-\mathrm{C}_{60}$ interactions [20,21], which gives qualitatively similar results. Furthermore, we expect other potentials, as for example proposed in [22-26], to corroborate our observations. However, when restricting the potential to a purely attractive van der Waals interaction only, we could not reproduce the experimental results.

The defined geometry is shown in figure 5(a), consisting of a plane of 45 molecules arranged according to the experimental observation. The used geometry includes a stacking fault, which leads to a bridging molecular row filling the gap at the domain boundary. This bridging row is formed by eight additional molecules, protruding the underneath layer. These protruding molecules are shown in darker color in figure 5(a).

To simulate constant height images of this system, we numerically calculate the normalized frequency shift $\gamma_{i, j}\left(z_{k}\right)$ in discrete steps at each lateral position $\left(x_{i}, y_{j}\right)$ and each vertical position $z_{k}$. Note that the normalized frequency shift $\gamma$ is directly proportional to $\mathrm{df}$ according to

$$
\gamma(z, A)=\frac{k_{0} A^{3 / 2}}{f_{0}} \mathrm{~d} f(z),
$$

where $A$ is the amplitude and $k_{0}$ is the stiffness of the cantilever [27]. To calculate the normalized frequency shift, we use a discretized formula of the normalized frequency shift as given by Giessibl [7]. This procedure including the used MATLAB code has been presented before [11]:

$$
\gamma_{i, j}\left(z_{k}\right)=\frac{A^{3 / 2}}{\pi} \sum_{n \in \mathbf{I}} k_{i, j}\left(z_{k}-(n-1) A\right) \sqrt{1-n^{2}} \times \Delta n
$$



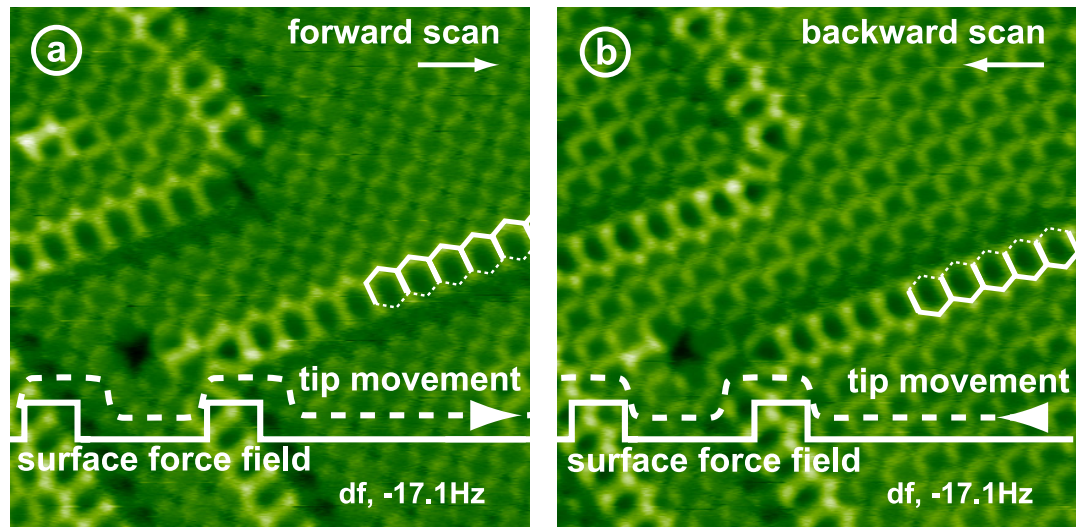

Figure 4. Comparison of forward and backward scans. Quasi-constant-height images with frequency shift setpoint given in the images. Frame size is $12 \mathrm{~nm} \times 12 \mathrm{~nm}$. (a) Forward scan (from left to right): the tip approaches the domain boundary from the left side, resulting in a domain boundary structure that appears open on the lower right side. (b) Backward scan showing a structure that is open on the upper left side. Combining both, forward and backward scans, the overall structure of the domain boundary reveals a hexagonal shape.

where $\mathbf{I}=\{m /(2 M) \mid m \in \mathbb{Z},-M \leqslant m \leqslant M\}$ and $M$ resembles the discretized amplitude. For all simulations we use a peak-to-peak amplitude of $2 A=20 \mathrm{~nm}$ as used in the experiments. The force gradients $k_{i, j}$ stem from a Morse potential (equation (3), whereby $E_{\text {bond }}, \sigma$ and $\kappa$ are the bonding energy, equilibrium distance and decay length) for which we used the parameters $\sigma=1.005 \mathrm{~nm}, \kappa=12.48 \mathrm{~nm}^{-1}$ and $E_{\text {bond }}=0.311 \mathrm{eV}$, as proposed in [28]. We want to stress that the simulations do not significantly depend on $\kappa$ and $E_{\text {bond }}$, especially the bonding energy only contributes as a prefactor in the normalized frequency shift.

$$
V_{\text {Morse }}(z, \kappa, \sigma)=E_{\text {bond }}\left(2 \mathrm{e}^{-\kappa(z-\sigma)}-\mathrm{e}^{-2 \kappa(z-\sigma)}\right) .
$$

The simulation results are shown in figures $3(\mathrm{~b})-(\mathrm{h})$. In figure 3(b) the normalized frequency versus distance curves are shown. The heights, at which the images in figures 3(c) to (h) were calculated, are indicated in figure $3(\mathrm{~b})$ by the corresponding symbols. The simulated images nicely reproduce the experimentally observed contrast formation. At distances far away from the surface, the $\mathrm{C}_{60}$ molecules appear bright, while upon approaching the tip towards the sample results in a contrast inversion. In figure $3(\mathrm{~h})$, the on top position of the $\mathrm{C}_{60}$ molecules appears dark while the area in between $\mathrm{C}_{60}$ molecules is imaged bright. Thus, this simulation confirm that the experimentally observed contrast is due to change in tip-sample interaction at different tip-sample distances. In particular, we can conclude that the used slow feedback loop settings do not influence the qualitative behavior, as our true constant height simulation results show an excellent agreement with the experimental results.

Besides the images, our simulations also confirm the experimentally observed $\mathrm{d} f(z)$ curves in a sense that we can reproduce the order of the crossing point in the three curves at positions $\mathrm{A}, \mathrm{B}$ and $\mathrm{C}$. We see, however, that the simulated curves deviate from the experimental ones as the simulated $\mathrm{d} f(z)$ curves at position $\mathrm{C}$ show negative slope at the crossing points of $\mathrm{A}$ and $\mathrm{B}$. This is in contrast to what is observed experimentally and illustrates the limits of this simple model. It has been reported before that the Morse potential fails to
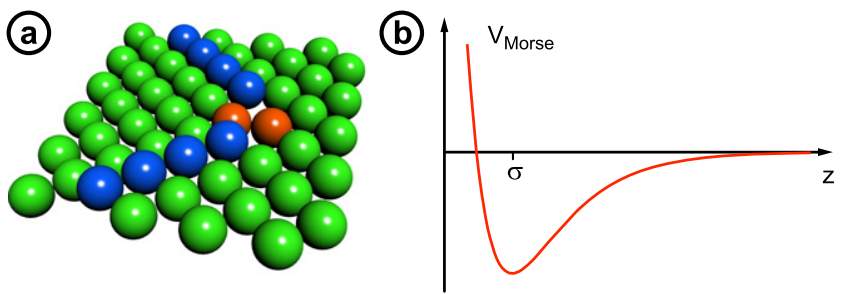

Figure 5. (a) Schematic of the simulated geometry. Each sphere corresponds to a single $\mathrm{C}_{60}$ molecule. Dark spheres correspond to the $\mathrm{C}_{60}$ layer, while the stacking fault molecules are colored medium grey. The protruding bridging row is given by spheres colored light grey. (b) Example of a Morse potential as used in the calculations.

model the repulsive regime as elastic contact forces have to be considered [29].

\section{Summary and conclusion}

The contrast formation of $\mathrm{C}_{60}$ molecules on the rutile $\mathrm{TiO}_{2}$ (110) was studied at different tip-sample distances. When imaging the molecules far away from the surface, the on top position appears bright while the area in between four molecules is imaged dark. Upon approaching the tip towards the surface, this contrast changes and is eventually inverted. We simulated the observed contrast inversion using a simple model system based on a Morse potential. These simulations reveal a qualitative picture of the contrast formation. Our study reveals that contrast inversion is observed due to crossing points in the frequency shift-distance curve. In the present case, these crossing points are not related to the onset of the repulsive regime. This study illustrates that an unambiguous identification of molecular positions within a molecular island using NC-AFM can require distance-dependent measurements of the contrast formation. The present system might represent a suitable starting point for in-depth theoretical considerations of contrast formation in NC-AFM. 


\section{Acknowledgments}

Financial support from the Deutsche Forschungsgemeinschaft (DFG) through an Emmy Noether grant and the integrated project PicoInside of the European Union is gratefully acknowledged.

\section{References}

[1] Joachim C, Gimzewski J K and Aviram A 2000 Nature 408 541-8

[2] Barth J V, Costantini G and Kern K 2005 Nature 437 671-9

[3] Kühnle A 2009 Curr. Opin. Colloid Interface Sci. 14 157-68

[4] Giessibl F J 1995 Science 267 68-71

[5] Reichling M and Barth C 1999 Phys. Rev. Lett. 83 768-71

[6] Barth C and Reichling M 2001 Nature 414 54-7

[7] Giessibl F J 2003 Rev. Mod. Phys. 75 949-83

[8] Barth C, Foster A S, Reichling M and Shluger A L 2001 J. Phys.: Condens. Matter 13 2061-79

[9] Mativetsky J M, Burke S A, Hoffmann R, Sun Y and Grütter P 2004 Nanotechnology 15 S40

[10] Lauritsen J V, Foster A S, Olesen G H, Christensen M C, Kühnle A, Helveg S, Rostrup-Nielsen J R, Clausen B S, Reichling M and Besenbacher F 2006 Nanotechnology 17 3436-41

[11] Rahe P, Bechstein R, Schütte J, Ostendorf F and Kühnle A 2008 Phys. Rev. B 77195410

[12] Foster A S, Hofer W A and Shluger A L 2001 Curr. Opin. Solid State Mater. Sci. 5 427-34
[13] Livshits A I, Shluger A L, Rohl A L and Foster A S 1999 Phys. Rev. B 59 2436-48

[14] Kobayashi K, Yamada H, Horiuchi T and Matsushige K 1999 Appl. Surf. Sci. 140 281-6

[15] Kobayashi K, Yamada H, Horiuchi T and Matsushige K 2000 Appl. Surf. Sci. 157 228-32

[16] Loske F, Bechstein R, Schütte J, Ostendorf F, Reichling M and Kühnle A 2009 Nanotechnology 20065606

[17] Zerweck U, Loppacher C, Otto T, Grafström S and Eng L M 2005 Phys. Rev. B 71125424

[18] Gritschneder S, Namai Y, Iwasawa Y and Reichling M 2005 Nanotechnology 16 S41

[19] Abe M, Sugimoto Y, Custance O and Morita S 2005 Appl. Phys. Lett. 87173503

[20] Girifalco L A 1991 J. Phys. Chem. 95 5370-1

[21] Pacheco J M and Ramalho J P P 1997 Phys. Rev. Lett. 79 3873-6

[22] GarciaRodeja J, Rey C and Gallego L J 1997 Phys. Rev. B 56 6466-9

[23] Guerin H 1998 J. Phys.: Condens. Matter 10 L527-32

[24] Hasegawa M, Nishidate K, Katayama M and Inaoka T 2003 J. Chem. Phys. 119 1386-96

[25] Li M 1999 Ann. Phys. 278 1-9

[26] Wulf K, Schiebel P, Prandl W, Paulus W and Heger G 1999 J. Phys.: Condens. Matter 11 2493-501

[27] Giessibl F J 1997 Phys. Rev. B 56 16010-5

[28] Feng-Ling L 2002 Acta Phys. Chim. Sin. 18 967-72

[29] Hölscher H, Schwarz A, Allers W, Schwarz U D and Wiesendanger R 2000 Phys. Rev. B 61 12678-81 